\title{
THE MOST COMMON GEOMETRIC AND SEMANTIC ERRORS IN CITYGML DATASETS
}

\author{
F. Biljecki ${ }^{\text {a, },}$ H. Ledoux ${ }^{\text {a } *}$, X. Du ${ }^{\text {a }}$, J. Stoter ${ }^{\text {a }}$, K. H. Soon ${ }^{\text {b }}$, V. H. S. Khoo ${ }^{\text {b }}$ \\ a 3D Geoinformation, Delft University of Technology, Delft, The Netherlands — (f.biljecki, h.ledoux, x.du-2, j.e.stoter)@ tudelft.nl \\ ${ }^{\mathrm{b}}$ Singapore Land Authority, Singapore — (soon_kean_huat, victor_khoo)@sla.gov.sg
}

KEY WORDS: CityGML, 3D city models, Quality control, Validation, Error

\begin{abstract}
:
To be used as input in most simulation and modelling software, 3D city models should be geometrically and topologically valid, and semantically rich. We investigate in this paper what is the quality of currently available CityGML datasets, i.e. we validate the geometry/topology of the 3D primitives (Solid and MultiSurface), and we validate whether the semantics of the boundary surfaces of buildings is correct or not. We have analysed all the CityGML datasets we could find, both from portals of cities and on different websites, plus a few that were made available to us. We have thus validated $40 \mathrm{M}$ surfaces in $16 \mathrm{M} 3 \mathrm{D}$ primitives and $3.6 \mathrm{M}$ buildings found in 37 CityGML datasets originating from 9 countries, and produced by several companies with diverse software and acquisition techniques. The results indicate that CityGML datasets without errors are rare, and those that are nearly valid are mostly simple LOD1 models. We report on the most common errors we have found, and analyse them. One main observation is that many of these errors could be automatically fixed or prevented with simple modifications to the modelling software. Our principal aim is to highlight the most common errors so that these are not repeated in the future. We hope that our paper and the open-source software we have developed will help raise awareness for data quality among data providers and 3D GIS software producers.
\end{abstract}

\section{INTRODUCTION}

In recent years, several cities around the world have released their 3D city models (3DCMs) as open datasets, and until recently they have been used mostly for visualisation. Such datasets are now being increasingly used as input for many different applications (Biljecki et al. 2015c). For example, they can help to assess the impact that environmental factors, such as noise (Stoter et al. 2008), wind (Janssen et al. 2013), and temperature (Lee et al. 2013, Hsieh et al. 2011), have on the citizens. Along with their increasing usage for spatial analyses, the topic of their quality and consistency has attracted attention, as several researchers started to recognise the importance of their validity (Agugiaro 2016, Bruse et al. 2015).

In general, to be used for advanced applications, 3DCMs need: (1) that their geometries are free of errors such as part of a roof missing, a bridge not connected to the shore, two houses slightly overlapping, houses "floating" a few centimetres above the ground, etc.; (2) to have rich semantic information. However, as several have observed (Steuer et al. 2015; Alam et al. 2014, Mulder. 2015, Stadler and Kolbe 2007; Pédrinis et al., 2015), 3DCMs are often plagued with errors and they do not always contain semantics, which, as a result, can have consequences because most simulation and analysis software assume that the input is valid (McKenney 1998).

The consistency of 3D city models has recently been increasingly investigated, and the OGC Quality Interoperability Experiment has been launched as solely devoted to this issue in CityGML models (OGC, 2016). Four aspects of data quality were studied and multiple software packages were developed to (partially) validate the following aspects: (1) schema conformance; (2) geometry (with a focus on solids); (3) semantics (with a focus on buildings); (4) conformance requirements (translation of requirements stated in natural language into verifiable functions).

\footnotetext{
${ }^{*}$ Corresponding authors. These authors contributed equally.
}

Related work considers additional aspects, e.g. resolvability of XLinks; application-specific rules, such as that a building is required to have a ground floor to form a volume (which is, surprisingly, not mandatory in CityGML); and the redundancy of the dataset, i.e. multiple identical geometries which are not re-used van Walstijn 2015, Biljecki et al. 2015b).

When inconsistencies are found, the only solution at this moment is to spend a substantial amount of hours manually repairing the data. There are ongoing efforts to automatically repair 3D models, see for instance Zhao et al. (2013), Alam et al. (2014) and Zhao et al. (2014). However, these only repair specific problems and have not been shown to be able to repair satisfactorily complex cases.

Since validation of 3D GIS datasets is a relatively new topic, there has not been a large-scale validation study on the current status of the quality of 3D city models. In this paper we bridge this gap, and attempt to answer a simple question: what is the geometric and semantic quality of the 3DCMs stored in CityGML, and what are the most common errors they contain? In Section 2 and Section 3 we elaborate on the validation method, and we validate all the 3DCMs we could obtain, both from portals of cities and on different websites, plus a few that were made available to us. We have validated 37 datasets from different sources and of different lineage, containing numerous buildings/features. Because buildings are the most prominent thematic feature of 3DCMs, we focus on them. However, most of the developed methodology is applicable to other thematic features as well. The quality of each is assessed by focusing on a subset of the aforementioned aspects (those that can be assessed and for which there are reliable tools/implementations):

1. Validation of the geometries in each of the 3DCM files by testing whether they comply to the definitions in the international standard ISO19107 (ISO, 2003). The rules we used and their meaning is further explained in Section 2 
2. Validation of the semantic information, if any, attached to the surfaces of the buildings in the 3DCMs. We explain our methodology in Section 3

3. Schema validation, i.e. is the XML of the files valid against the schema of CityGML? For the purpose of this paper, we consider this a "solved problem" that needs no further explanation since several tools exists.

In Section 4, we present, discuss, and analyse the errors we found and try to explain their source. Based on our experiments, we make in Section 5 recommendations that practitioners and data producers can follow when constructing 3DCMs. It should be pointed out that the purpose of this paper is not to shame the data producers, some of the models we validate date back to several years and producing error-free models was certainly not a priority. We aim at highlighting the most common errors that exist in 3DCMs, so that these are not repeated in the future, and we hope that our paper will help to raise awareness for 3D data quality among data providers. Furthermore, the developed validation software is released as open-source software, which enables data producers to check the produced datasets with the same methodology as used in this paper, in order to fix errors before the delivery of the data, and/or to include similar workflows in modelling software.

\section{VALIDATION OF THE GEOMETRY OF INDIVIDUAL FEATURES}

\subsection{Geometric primitives}

CityGML (OGC |2012), and GML (OGC, 2007), use the ISO19107 geometric primitives for representing the geometry of its objects (ISO 2003): a 0D primitive is a GM_Point, a 1D a GM_Curve, a 2D a GM_Surface, and a 3D a GM_Solid. A $d$-dimensional primitive is built with a set of $(d-1)$-dimensional primitives, e.g. a GM_Solid is formed by several GM_Surfaces, which are formed of several GM_Curves, which are themselves formed of GM_Point. While the ISO19107 primitives do not need to be linear or planar, i.e. curves defined by mathematical functions are allowed, CityGML uses a subset of ISO19107, with the following two restrictions: (1) GM_Curves can only be linear (thus only LineStrings and LinearRings are used); (2) GM_Surfaces can only be planar (thus Polygons are used).

\subsection{Aggregates \& Composites}

Following ISO19107, in GML and CityGML geometric primitives can be combined into either aggregates or composites.

An aggregate (class gml:_AbstractGeometricAggregate) is an arbitrary collection of primitives of same dimensionality that is simply used to bundle together geometries. GML (and CityGML) has classes for each dimensionality (Multi*), the most relevant one in our context is MultiSurface that is often used in practice to represent the geometry of a building. An aggregate does not prescribe any topological relationships between the primitives.

A composite of dimension $d$ is a collection of $d$-dimensional primitives that form a $d$-manifold, which is a topological space that is locally like a $d$-dimensional Euclidean space $\left(\mathbb{R}^{d}\right)$. The most relevant example in our context is a CompositeSurface: it is a 2-manifold, or, in other words, a surface embedded in $\mathbb{R}^{3}$. An obvious example is the surface of the Earth, for which near to every point the surrounding area is topologically equivalent to a plane. This implies that the surfaces part of a composite are not allowed to overlap and/or to be disjoint. Furthermore, if we store a CompositeSurface in a data structure, each edge is guaranteed to have a maximum of two incident surfaces, and around each vertex the incident faces form one 'umbrella'.

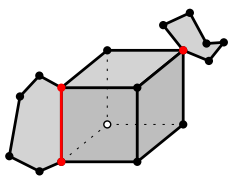

(a)

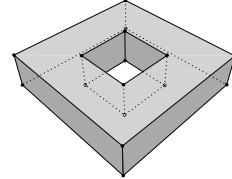

(b)

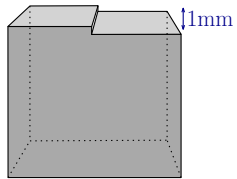

(c)
Figure 1: Some examples of Solids. (a) An invalid one because both one edge and one vertex are non-manifold (the red ones). (b) A 'squared torus' is modelled with one exterior boundary formed of ten surfaces. Notice that there are no interior boundary. (c) All eight points of the top surface have their $z$ coordinate within $1 \mathrm{~mm}$, but the polygon cannot be considered planar, and thus the solid would invalid (see Sec. 2.4).

A CompositeSurface that is closed (there should not be 'holes' in the surface, it should be 'watertight') and orientable is referred to as a Shell (see Figure 2). Shells are used to define the boundaries of a Solid. In Figure 2, the Solid has two boundaries: an exterior one (the cube in grey) and one interior one (the cube in orange), which defines a 'void' in the solid. A Solid can have an infinity of interior boundaries, or none. Observe that a cavity is not the same as a hole in a torus (a donut) such as that in Figure $1 \mathrm{~b}$ it can be represented with one exterior boundary having a genus of 1 and no interior shell. Interior boundaries in surfaces are possible, simple LOD1 buildings having for instance an inner yard require them, as Figure $1 \mathrm{~b}$ shows.

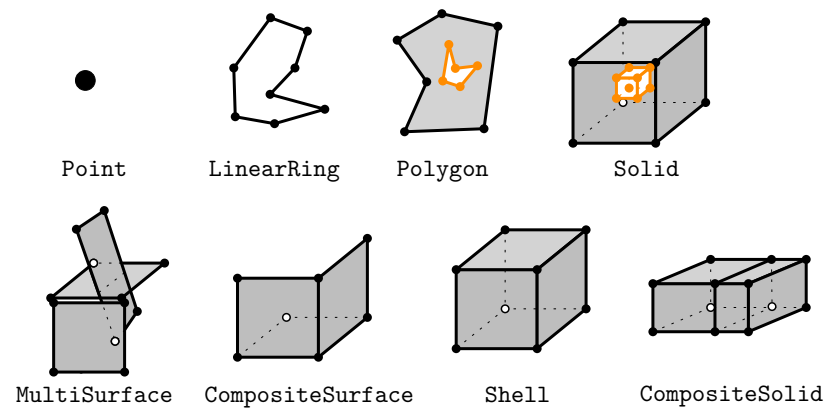

Figure 2: Some of the CityGML primitives, including aggregates and composites. Orange primitives are those representing inner boundaries. The Shell is not a class in GML, but it is implied when a CompositeSurface is used to define the boundary of a Solid.

\subsection{How we validate the geometry of primitives}

For our experiments, we used the validation methodology as described in Ledoux (2013) for solids, which is ISO19107-compliant. Figure 3 shows the error codes that can be reported, for different 3D primitives. The methodology is hierarchical, i.e. a solid is validated by validating all primitives of lower-dimensionalities first, starting with LinearRings. This means that if one or more Polygons of a Solid is invalid, the validation process will be stopped before the primitives of a higher are validated (to avoid errors causing false errors in primitives of higher-dimensionalities). One invalid Solid would therefore not be reported as invalid with errors 202 and 301, since the validation would stop at the level of the Polygon (level 2xx). All primitives at a given level are however validated. 


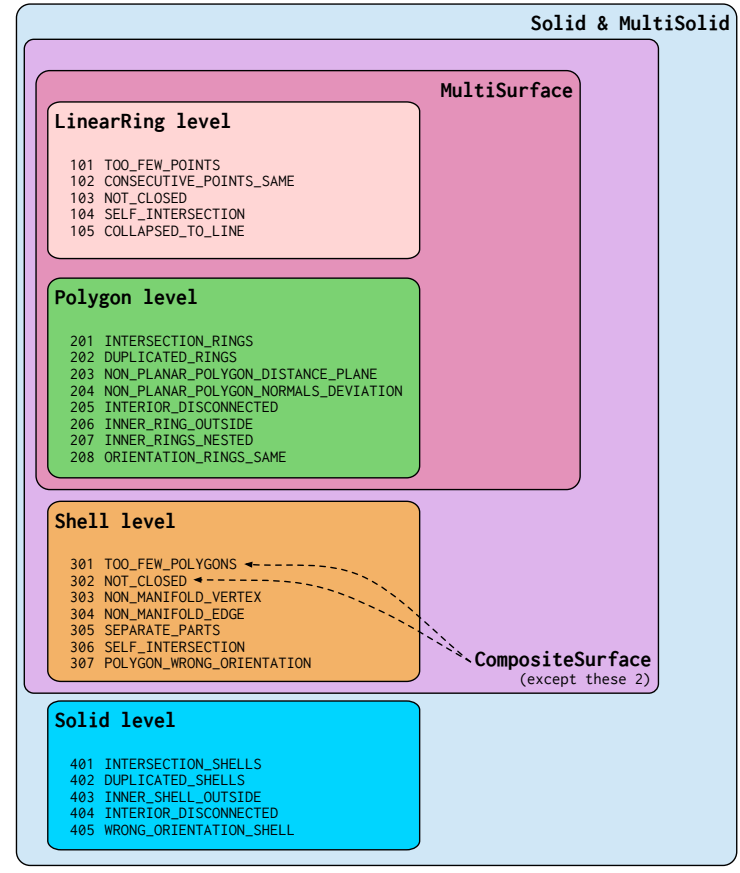

Figure 3: Error codes for the validation of geometric primitives.

For this paper, we are focusing only on buildings. As a consequence: (1) interior boundaries in solids are ignored. This implies that a solid has only one shell representing its exterior boundary; (2) we focus on the two representations for Buildings in CityGML: MultiSurfaces or Solid/CompositeSolids. We validate MultiSurface primitives by using a subset of the methodology for Solids: validating an aggregate simply implies validating individually each primitive in the collection. We do not explicitly validate CompositeSurface. Also, we do not validate CompositeSolids since we do not have access to a software implementation that would allow us to verify the topological relationships between different solids. This is a shortcoming of our current result, since CompositeSolids are sometimes used to represent complex buildings (e.g. with several BuildingPart). We show examples in Section 4 However, we validate MultiSolid primitives, if present, by validating each Solid separately.

\subsection{Tolerances for coordinates and planarity}

Geometries modelled in CityGML store amazingly very little topological relationships. For instance, all six surfaces of a cube are often stored independently. This means that the coordinates $(x, y, z)$ of a single point (where three polygons "meet") is stored three times. It is possible that these three vertices are not exactly at the same location, e.g. $(0.01,0.5,1.0),(0.011,0.49999$, $1.00004)$ and $(0.01002,0.5002,1.0007)$, and that would create problems when validating since there would for example be tiny cracks/overlaps in the cube. We therefore use a snapping tolerance on the input coordinates for each primitive, this value is $1 \mathrm{~mm}$.

While both ISO19017 and CityGML mention that each Surface must be planar (i.e. all its points, used for both the exterior and interior rings, must lie on a plane), the concept of tolerance is not mentioned. We believe this is a shortcoming, and thus use two concepts that were defined during the OGC CityGML QIE (OGC) 2016):

1. the distance between every point forming a polygon and a plane is less than $\epsilon_{1}$, a given tolerance (e.g. $1 \mathrm{~mm}$ ). This plane should be a plane fitted with least-square adjustment.
2. the distance between every point forming a polygon and all the planes defined by all possible combinations of 3 noncollinear points is less than $\epsilon_{1}$.

The second requirement is to ensure that cases such as that in Figure $1 \mathrm{c}$ are detected. From algorithmic point of view, the definition is not very efficient, but in practice it can be implemented with a triangulation of the polygon (any triangulation): the orientation of the normal of each triangle must not deviate more than than a certain user-defined tolerance $\epsilon_{2}$ (e.g. 1 degree).

\section{VALIDATION OF THE SEMANTICS OF BUILDINGS}

In CityGML, each of the surfaces used to represent a building can be a semantic class (BoundarySurface), which defines its realworld meaning. Depending on the LOD, a BoundarySurface, can be one of nine classes. In this paper, we focus on the five possible ones for LOD2 buildings; these are shown in Figure 4

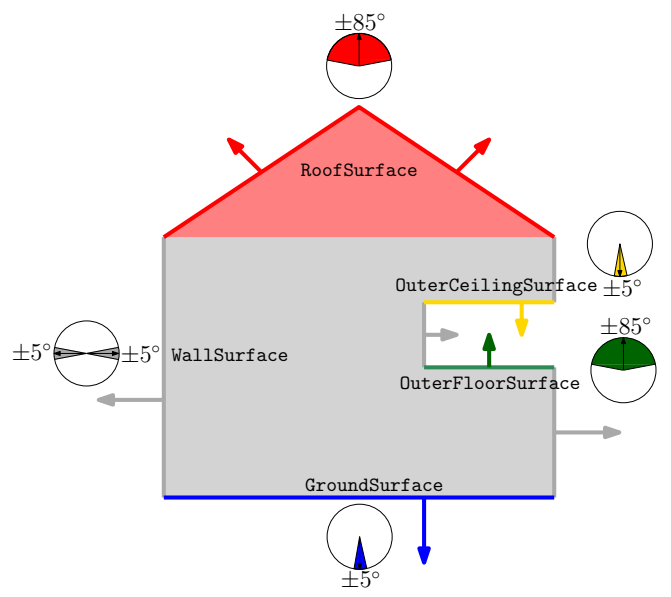

Figure 4: The semantic classes for buildings that we validate, and the angles and tolerances we use.

While storing the semantic information of each surface is not mandatory, CityGML encourages it and sees it as one of its core principle, since, as Stadler and Kolbe (2007) demonstrate, it can be beneficial for several applications. As can be seen in our experiments, the semantics of LOD2 models is often stored in practice.

While it is impossible to validate with $100 \%$ certainty the semantic of the surfaces of a building, we can infer it from the orientation of a surface. We follow the work of others (Boeters et al. 2015, Diakité et al. 2014, OGC 2016), and compare the direction of the normal of the surface to a reference for each class, which we have defined in Figure 4

Because calculating the normal of a surface that is invalid (e.g. if it self-intersects, is not planar, etc.) might not be possible, for each surface we perform a linear least squares fitting (with a plane), and use the normal of that plane. Only when an invalid surface has less than 3 points we skip the normal calculation. A surface which normal is not in the range defined in Figure 4 is regarded as invalid. Observe that doors, windows and closure surfaces are not considered in this paper.

\section{ANALYSIS AND RESULTS}

\subsection{Datasets}

We have validated 37 datasets from 9 countries, containing $3.6 \mathrm{M}$ buildings represented with $40 \mathrm{M}$ polygons. The datasets have 
been obtained from open data portals of cities and universities, demo datasets from companies, and a few have been procedurally generated to add to the diversity. In order to focus on the discovered inconsistencies, and instead of giving an impression of blaming specific sources and producers, we do not identify the datasets in the analysis. We have merely listed the sources in the Acknowledgements.

\subsection{Tools}

For the validation of the geometry and semantics we have used the open-source software val3dity, as Ledoux (2013) describes. The schema validation was implemented in a script using Xerces, and we implemented ourselves the semantic validation. All the software is released as open-source ${ }^{1}$ under a GPL licence. Hence our approach can be easily reproduced.

\subsection{Used quality metrics}

Since the result of a validation is binary (something is valid or not), we express errors as percentages. This is also because there is a great variation of characteristics of the datasets (some datasets contain a few buildings, while others more than one hundred thousand). However, because of the different sizes of datasets and varying structure (e.g. $20 \%$ invalid solids is not the same as $20 \%$ invalid buildings; this is later explained in Figure 6), we focus on expressing the errors on the most granular level: percentage of valid primitives for geometry, and percentage of valid surfaces when it comes to the semantics. For the schema validation, we state whether the files (sometimes several files are used for a given city) are valid against the schema; we have noticed that in multi-file datasets either all the files are valid or invalid.

\subsection{Results}

We have listed the results of the three analyses in Table 1 The results show a substantial variation in the quality of the analysed datasets. Some datasets are almost valid, while in some almost all primitives are invalid. Perfectly valid datasets are very rare, as LOD1 models contained at least $0.1 \%$ to $0.5 \%$ errors. As partially visible in Table 1 we have found that no two datasets are alike when it comes to errors (see also Fig. 5). This is likely caused by different software packages used for modelling, different workflows used to produce the models, and the different companies with different internal procedures for quality assurance. This prevents us to generalise a common conclusion about the quality, and makes it difficult to come up with a list of common errors. While one dataset is infested with one type of error, such error is not found at all in another one.

It should be noticed that models in LOD1 are almost all valid, except a few isolated exceptions; the errors are mostly duplicated vertices. Also, some datasets have an invalid syntax (schema), which meant that we could not parse it to validate it. Observe that for many invalid ones we could still parse the files and validate their geometric primitives and their semantics; the errors were located in parts that were not relevant to our task.

Another general observation is that the geometric and semantic errors are not correlated (see Fig. 6). While some datasets are full of geometric errors, they fare much better in the semantic aspect. Furthermore, the size of the dataset appear not to have an influence on the errors-we had expected that smaller datasets might have been prepared more carefully. But it turns out that is not the case.

1 https://github.com/tudelft3d/val3dity and http: //geovalidation.bk.tudelft.nl

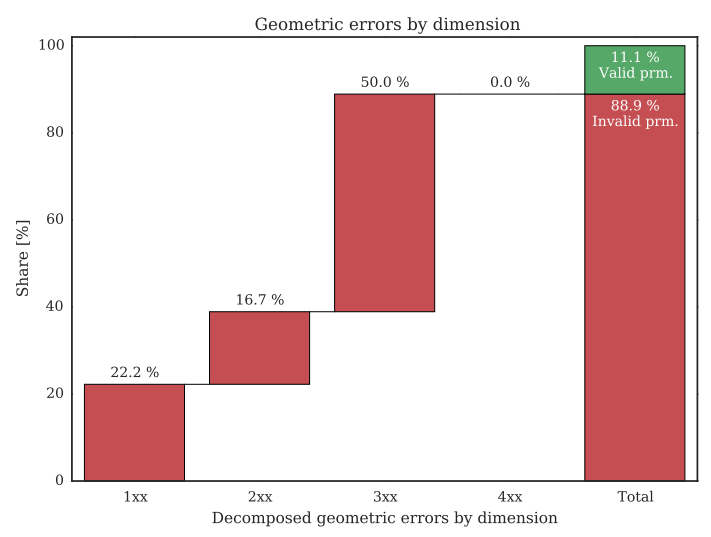

Figure 5: Decomposition of the geometric errors into 4 levels (example an LOD2 with solids). Each of the datasets has a unique configuration of errors preventing a common conclusion about the quality of CityGML datasets. Example of dataset \#27.

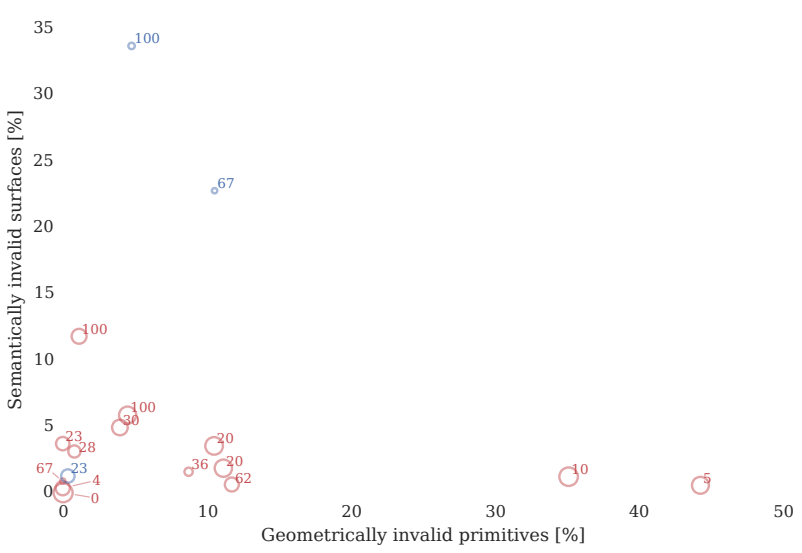

Figure 6: Joint spatio-semantic quality plot for semantically structured 3D models in our analysis. The numbers represent the percent of semantically invalid buildings, in contrast to the percent of invalid surfaces (on the y axis) - e.g. notice that one dataset (\#16) contains just 1\% invalid surfaces, but it seems that the error is prevalent in most $(62 \%)$ buildings. Such difference between the $\%$ of invalid surfaces in contrast to the $\%$ of invalid buildings illustrates a challenge in defining quality metrics. The colour refers to the LOD (2-red, 3-blue), and the diameter of the circle indicates the size of the dataset. 
Table 1: Results of the validation of the test datasets. The share of errors is expressed in per cents.

\begin{tabular}{|c|c|c|c|c|c|c|c|c|c|}
\hline \multirow[t]{2}{*}{ Level of detail } & \multirow[t]{2}{*}{ Primitive } & \multirow[t]{2}{*}{$\mathrm{ID}^{(\mathrm{a})}$} & \multicolumn{5}{|c|}{ Geometric validation } & \multirow[t]{2}{*}{ Semantics $^{(f)}$} & \multirow[t]{2}{*}{ Schema } \\
\hline & & & $1 \mathrm{xx}$ & $2 \mathrm{xx}$ & $3 x x$ & $4 \mathrm{xx}$ & Total $^{(\mathrm{b})}$ & & \\
\hline \multirow[t]{9}{*}{ LOD1 } & Solid & 1 & 0 & 0 & 0 & 0 & 0 & - & $x$ \\
\hline & & 2 & 0 & 0 & 0 & 0 & 0 & - & $\checkmark$ \\
\hline & & 3 & 0 & 0 & 0 & 0 & 0 & - & $\checkmark$ \\
\hline & & 4 & 0 & 0 & 0 & 0 & 0 & - & $\checkmark$ \\
\hline & & 5 & 0 & 0 & 0 & 0 & 0 & - & $x$ \\
\hline & & 6 & 0 & 0 & 0 & 0 & 0 & - & $x$ \\
\hline & & 7 & 0 & 0 & 0 & 0 & 0 & - & $\checkmark$ \\
\hline & & 8 & 0 & 0 & 0 & 0 & 0 & - & $\checkmark$ \\
\hline & & 9 & 0 & 0 & 0 & 0 & 0 & - & $x$ \\
\hline \multirow[t]{12}{*}{ LOD2 } & MultiSurface & 10 & 1 & 4 & - & - & 5 & (e) & $\checkmark$ \\
\hline & & 11 & 0 & 0 & - & - & 0 & 0 & $\checkmark$ \\
\hline & & 12 & 2 & 21 & - & - & 23 & 45 & $\checkmark$ \\
\hline & & 13 & 10 & 2 & - & - & 12 & 4 & $x$ \\
\hline & & 14 & 0 & 1 & - & - & 1 & 12 & $\checkmark$ \\
\hline & & 15 & 0 & 9 & - & - & 9 & 2 & $\checkmark$ \\
\hline & & 16 & 4 & 8 & - & - & 12 & 1 & $x$ \\
\hline & & 17 & 5 & 0 & - & - & 5 & 5 & $x$ \\
\hline & & 18 & 0 & 0 & - & - & 0 & 4 & $x$ \\
\hline & & 19 & 0 & 0 & - & - & 0 & 1 & $x$ \\
\hline & & 20 & 0 & 4 & - & - & 4 & 6 & $x$ \\
\hline & & 21 & 0 & 1 & - & - & 1 & 3 & $x$ \\
\hline \multirow[t]{6}{*}{ LOD2 } & Solid & 22 & 0 & 42 & 58 & 0 & 100 & - & $\checkmark$ \\
\hline & & $23^{(\mathrm{c})}$ & - & - & - & - & - & - & $x$ \\
\hline & & 24 & 0 & 31 & 1 & 3 & 35 & - & $x$ \\
\hline & & 25 & 4 & 0 & 16 & 2 & 22 & - & $\checkmark$ \\
\hline & & $26^{(\mathrm{c})}$ & - & - & - & - & - & - & $x$ \\
\hline & & 27 & 22 & 17 & 50 & 0 & 89 & - & $\checkmark$ \\
\hline \multirow[t]{4}{*}{ LOD2 } & MultiSurface & 28 & 0 & 42 & 1 & 1 & 44 & 0 & $\checkmark$ \\
\hline & and Solid ${ }^{(\mathrm{d})}$ & 29 & 2 & 35 & 54 & 0 & 92 & 4 & $\checkmark$ \\
\hline & & 30 & 0 & 10 & 0 & 1 & 11 & 2 & $\checkmark$ \\
\hline & & 31 & 0 & 0 & 0 & 0 & 0 & 0 & $\checkmark$ \\
\hline \multirow[t]{5}{*}{ LOD3 } & MultiSurface & 32 & 2 & 13 & - & - & 15 & 54 & $\checkmark$ \\
\hline & & 33 & 6 & 5 & - & - & 11 & 23 & $\checkmark$ \\
\hline & & 34 & 8 & 10 & - & - & 19 & 45 & $\checkmark$ \\
\hline & & 35 & 5 & 0 & - & - & 5 & 34 & $\checkmark$ \\
\hline & & 36 & 0 & 0 & - & - & 0 & 1 & $x$ \\
\hline LOD4 & Solid & 37 & 0 & 0 & 3 & 0 & 3 & 68 & $\checkmark$ \\
\hline \multicolumn{10}{|c|}{$\begin{array}{l}\text { (a) Anonymised dataset ID. } \\
\text { (b) The decomposed errors may not sum up to the totals due to rounding. Most of LOD1 datasets are not } 100 \% \text { valid, they } \\
\text { are rather between } 99.5 \% \text { and } 99.9 \% \text { valid. } \\
\text { (c) The dataset could not be read by our software due to errors while parsing the GML input. } \\
\text { (d) These datasets contain both the Solid and MultiSurface (usually realised through the XLink mechanism). } \\
\text { (e) Dataset with MultiSurfaces but without semantic information. } \\
\text { (f) Our approach for the semantic validation falls short with some datasets (further explained in Fig. } 15 \text {, and high values } \\
\text { should be ignored. }\end{array}$} \\
\hline
\end{tabular}

\subsection{Common errors}

We have examined the errors in the datasets and the first observation is that errors appear to be random in some datasets, while in some datasets they are of a systematic nature. For instance, Fig. 7 shows a dataset with one random error: a missing wall from a building. On the other hand, we have encountered repetitive errors that could have been caused by software or by the lack of guidelines. For instance, wrong orientation of linear rings. We came up with a list of errors that can be found in multiple datasets and describe them in the continuation, in no particular order.
4.5.1 Geometries not properly snapped A common cause for invalid solids (Error 3xx) are geometries that are not snapped together, which causes surfaces to intersect and/or to result a nonwatertight solid. One example is shown Figure 8 Observe that the invalid solids which we have encountered often look correct with a visual inspection, and only by zooming in one can detect these. As explained in Section 2.4 we used a snapping tolerance of $1 \mathrm{~mm}$ on the input coordinates. Thus for such errors the vertices are further than this apart.

4.5.2 Invalid semantics Surfaces with erroneously assigned semantics are easy to analyse - they can be coloured differently 


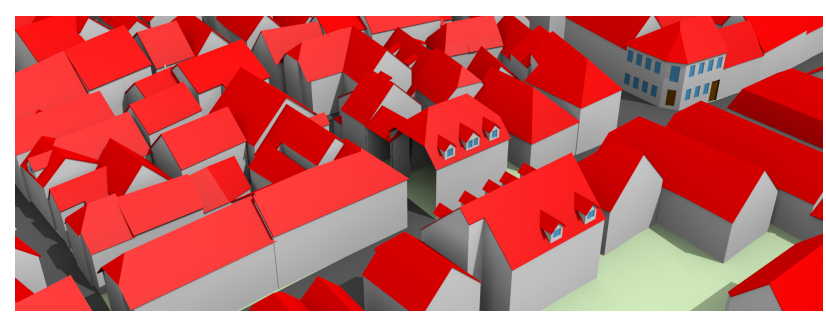

Figure 7: An example of a seemingly random error: missing wall polygons from a building, causing invalid solids. This error is likely caused by the conversion of the dataset from another format, during which the generation of the polygons failed.

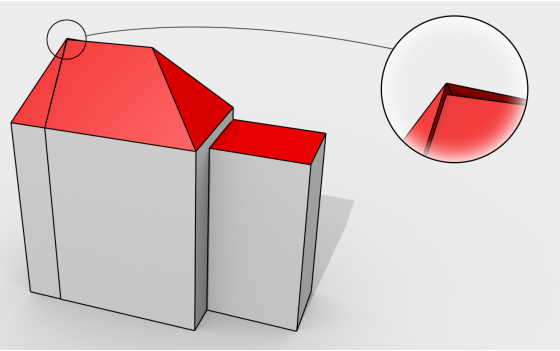

Figure 8: Not snapped geometries are the usual cause of invalid solids.

while modelling. Despite this, we have detected a fair share of semantically incorrect surfaces. However, we have not encountered a pattern. Hence we cannot focus on a particular type of such errors. Fig. 9 shows an interesting example of a semantically invalid surface.

Most of the datasets are semantically valid, and we suspect that this is because in many cases the semantic information was added as a post-processing by using the exact same method we use for validation (by using the normal of the surfaces).

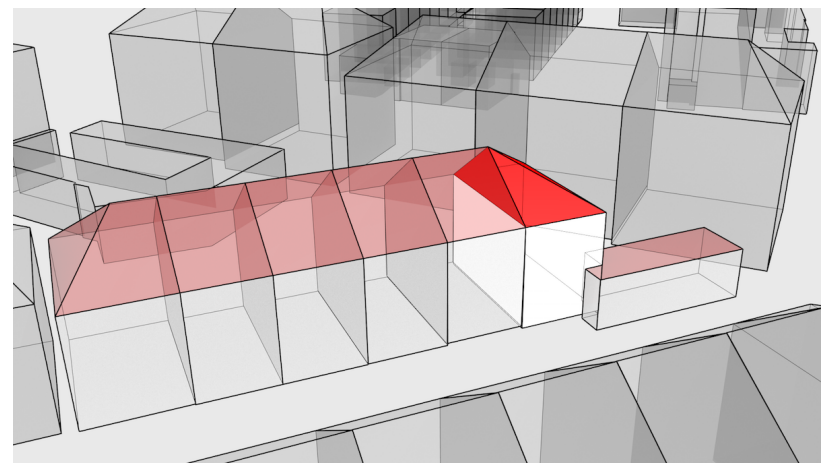

Figure 9: This example illustrates two inconsistencies: (a) a RoofSurface used to store a vertical interior wall (right end of the building); and (b) a building complex decomposed into multiple buildings and separated by duplicated WallSurfaces. While the former error is normally noticeable by a visual inspection while modelling, it likely went unnoticed because it is obstructed, being inside the building.

4.5.3 Non-planarity As explained in Section 2.4 we have used two methods to detect non-planar polygons, each having their own error code (203 and 204). The results show that there are many non-planar polygons (e.g. dataset \#28 has more than $40 \%$ invalid polygons due to planarity issues), and we would highlight this as the most common error in all the datasets we

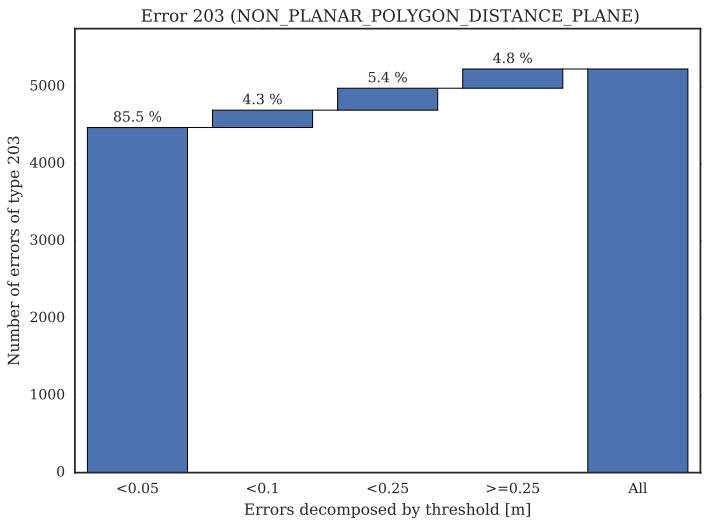

Figure 10: Decomposition of the planarity errors (Error 203) into the distance from the fitted plane. Most of the errors are due to deviations of a few centimetres. Example from dataset \#24.

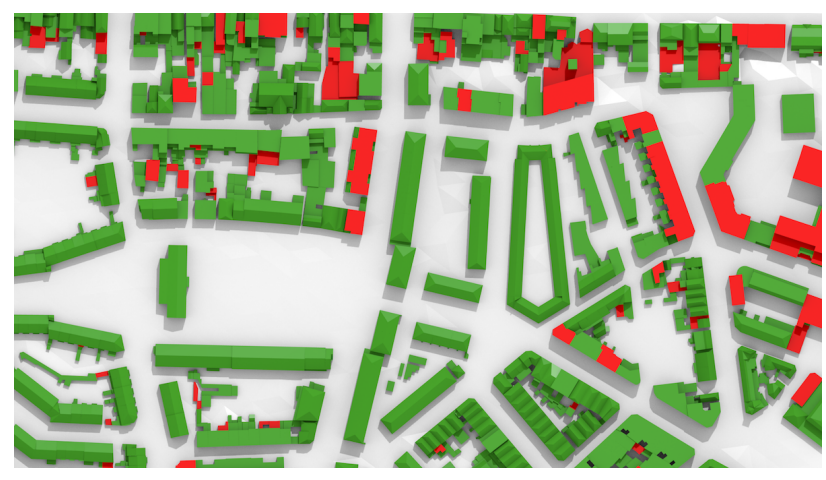

Figure 11: A common error we have noticed is the wrong orientation of semantic surfaces stored in MultiSurface. In this example, the correctly oriented Roof Surfaces are shown in green, and those with the inverted orientation (errors) are shown in red.

tested. Non-planar polygons may cause issues when calculating their area, and in visualisation (texturing), and can-if the deviation is very large-cause intersections with other surfaces in a solid. We have decomposed the errors related to planarity by their severity (in case of the Error 203: by the deviation from the fitted plane). The decomposed errors show that most planarity errors are caused by deviations of just a few centimetres (Fig. 10), which is perhaps not an issue for many applications, but is nonetheless invalid according to the internal standard.

This is probably the reason why they have not been noticed, as it is visually impossible to detect them. Furthermore, the large deviations visible in the plot are mostly found in very large polygons (roof of a $50 \mathrm{~m}$ long warehouse), where this inconsistency is hard to notice, even if gross.

4.5.4 Polygon orientation Many polygons in a few datasets have been found to be incorrectly oriented (see Fig. 111. A likely cause of these errors is the fact that many software packages do not distinguish the orientations. This error causes solids to be invalid even when the polygons comprising it make it watertight. If stored as a MultiSurface, then the building would be valid (albeit less useful for spatial analysis). 


\section{DISCUSSION, RECOMMENDATIONS \& FUTURE WORK}

\subsection{General observations}

In our analysis we have encountered many different errors, of which some are common across multiple datasets. We suspect that data producers perform visual quality checks by removing errors that can be caught with a manual inspection. This leaves the following reasons why some datasets have a considerable number of errors: (1) practitioners are simply not aware of possible errors and of the international standards; (2) the modelling software has no validation support; and (3) the produced data works in the applications intended for the data (which often appears to be visualisation), hence the errors which may influence other applications are simply undetected or ignored.

The analysis of the datasets shows some patterns interesting to note. First, it appears that datasets of a finer LOD are more prone to errors in comparison to their coarser counterparts; this is due to the complexity of the geometry. Datasets in LOD1 and synthetically generated datasets contain least errors, and performed best in our experiments. Second, specific modelling conventions resulted in gross errors of otherwise fine datasets. For instance, the dataset \#29 contains $92 \%$ of invalid solids. The reason behind this error is the modelling approach of assigning a joint GroundSurface to multiple building parts that are connected. Otherwise, most solids would be valid.

\subsection{Remedies}

These common errors could be solved by imposing constraints and/or using an ISO-compliant validator. Furthermore, advancing the topological functionalities of the software to prevent them would also greatly help (e.g. enable snapping). However, we are also under the impression that some of these errors are directly caused by software: some datasets have been produced in formats other than CityGML (e.g. IFC, DXF, DAE), and the conversion to CityGML introduced new errors.

In addition to modelling software, the errors may also depend on the data source that is used as input. Different data sources (e.g. building footprints, point clouds) dictate the information that can be observed by the modeller. For instance, when using the orthophoto, sometimes due to certain hidden surfaces (e.g. outer ceiling surface), overlaps are intentionally created between building surfaces to ensure that there are no gaps them. Such intention then becomes a topological error.

Despite advancements in the repair of dataset (Zhao et al. 2013. Alam et al. 2013, we deem that in the foreseeable future it is not realistic to expect a universal solution that would automatically repair data-it would rather be beneficial to focus on preventing errors while modelling. While such automatic solutions do manage to repair a fair share of 3D models, it is simply hard to account all cases which we have encountered, and we believe that manual repair will still dominate as the preferred method for healing 3D models.

\subsection{Impact on applications}

While our results show that many datasets contain a substantial amount of errors, their severity eventually depends on their intended use. Some of the errors have no influence on the application, while others may completely prevent their use. Therefore it is not only a matter of analysing the quantity of errors in 3DCMs but also of identifying those which has severe impact on the applications. For instance, using the data for visualisation purposes is likely less prone to errors than when using it for applications such as the simulation of the airflow in a city with computational fluid dynamics. In fact, the inability to use 3D city models for a specific purpose has been the main driving factor of some related work, e.g. Steuer et al. (2015), Mulder (2015), and Sindram et al. (2016) develop repair techniques specifically designed to overcome topological errors that prevent the computation of the volume of buildings.

Besides the geometric aspect, many applications are prone to semantic errors as well. For instance, the estimation of the solar potential of roof surfaces is susceptible to erroneous semantics of surfaces (e.g. a wall being mislabelled as a roof surface and accounted in the total area feasible for the installation of solar panels). While there is a great deal of investigating the propagation of error in GIS (e.g. see the overview in Biljecki et al. (2015a)), the propagation of errors described in this paper has not been investigated, and it is a topic for future work. This is especially important in the context of open data, since it is to be expected that they will be used in a wide range of purposes. As documented by several papers, e.g. see (Salimzadeh et al. 2016, Nouvel et al. 2015, Boeters et al. 2015, Kehl 2015), researchers show variable level of satisfaction about publicly available 3D data, depending on the used application.

It is important to note that besides the employment of models in spatial analyses, errors may influence other tasks such as storage in databases, maintenance of data, and conversion to other formats. For instance, non-planar polygons may present a problem in the triangulation, when converting CityGML models to computer graphics formats such as OBJ.

\subsection{Limitations, open questions, and future work}

The software we used does not cover all aspects of quality, hence not all errors have been detected. While manually inspecting the datasets we have realised that there are other types of inconsistencies which we recommend to investigate in future work. In the continuation, we list the most important ones.

One relevant CityGML conformance requirement-that we not consider in this paper-is the one concerned with Building and BuildingPart. The standard states that if a building is one homogeneous part it should be represented as one Building, but different BuildingPart should be used if the roof types or if the number of storeys differ, or if the addresses are different.

We found several questionable cases in our datasets, see for instance Figure 12 where a large building complex is modelled as one Building having several BuildingPart, while it should arguably be modelled with different buildings (at least from the European perspective). This example (along with the one in Fig. 13, also shows that the notion of a building varies between datasets. Our prototype can detect such cases by finding buildings with an

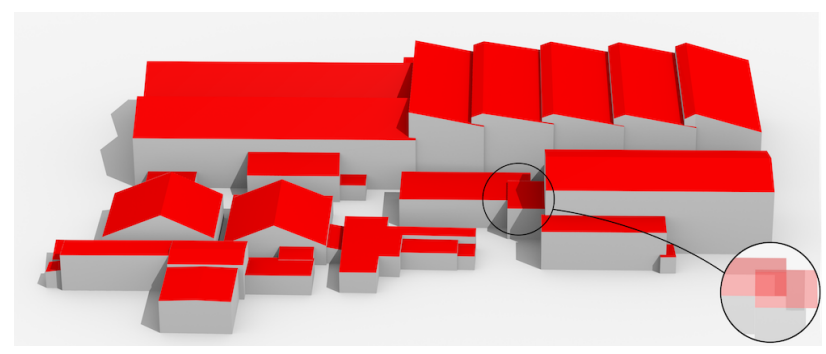

Figure 12: (Arguably) multiple buildings stored as one building with many building parts, which also overlap. 
unusually large number of polygons and of a large surface area, but this solution is not mature enough to carry out rigorous experiments (e.g. this approach flags large valid buildings such as cathedrals). Belussi et al. (2015) propose a solution based on OCL constraints, but their implementation does not support interior boundaries in polygons at this moment.

The previous figure doubles as another example of an idea for future work: overlap of features, which has not been covered at this moment. We have noticed that at several locations the BuildingParts are overlapping, which cause the building volume, if calculated by summing the volumes of the parts, to be larger than the true value.

This error is also illustrated in Fig. 13. where awnings (stored as MultiSurfaces) overlap with the facade (stored as a Solid), i.e. they are not clipped. As previously mentioned, we do not analyse the topological relationships between different objects, as this is a topic for future work. Such errors would have been prevented by more advanced modelling capabilities and constraints in the software.

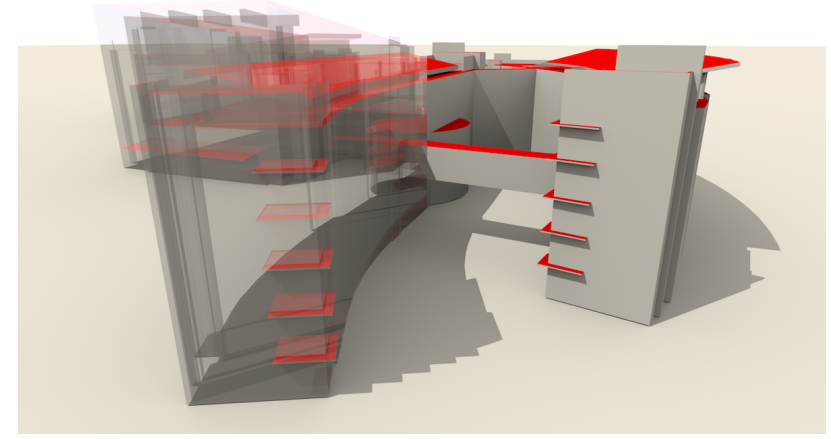

Figure 13: Outer surfaces protruding inside the walls.

The validation of the level of detail (LOD) in datasets presents an important idea for future work, for instance checking if the features have been acquired according to the LOD stated in the metadata. Fig. 14 shows an example of a dataset of a heterogeneous LOD. This is a simple case which can be detected because the buildings are stored in different CityGML LODs, but there are other examples in which a building is stored as an LOD3 but it is evident that it is an LOD2 because it contains no openings (for a related example compare the buildings in Fig. 7). This can involve developing methods to automatically detect the LOD of objects, based on related work in 2D (Touya and Brando-Escobar 2013), but it is hampered by the fact that there is a great variation between buildings when it comes to complexity, hence inferring their LOD may be complex. For example, a garage in LOD3 may be modelled as a simple block akin to an LOD1, and still be valid (Biljecki et al. 2016).

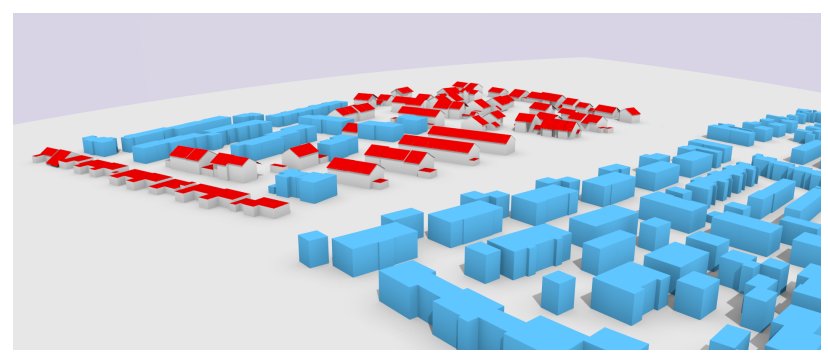

Figure 14: Inconsistent level of detail of the dataset.

The limitations of our method yields a number of false positives and false negatives, especially in the semantic validation (see Figs. 15 and 16 for examples). For instance, a shortcoming in the semantic validation is that we cannot distinguish between OuterFloorSurface and RoofSurface, since they are validated against the same range of angles (see Figure 4).

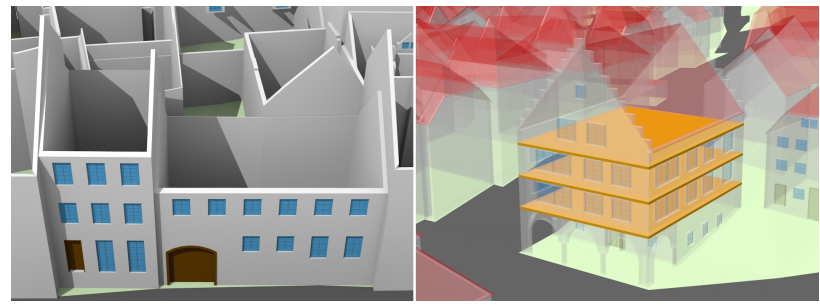

Figure 15: Examples of the shortcomings of our semantic validation. The left image shows a false negative-a WallSurface is modelled as a volume, and the tops have the normals pointing upwards resulting in an error. The render on the right shows a building with interior floors modelled as GroundSurface (in orange). This is a false negative because the error has not been detected by our normal-based approach. Oddly enough, the one GroundSurface which is supposed to be there is missing, but this omission has not been detected either. Both situations are likely caused by the IFC origin of the dataset, as in the conversion the geometry was not adapted to the structure expected by CityGML. Making the semantic validation more intelligent is a priority for future work.
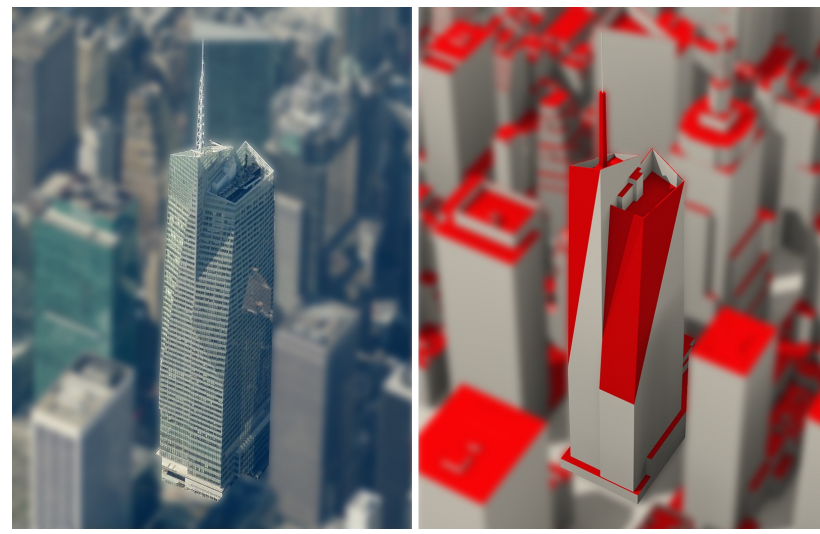

Figure 16: Example of an uncertain situation: a wall modelled as a RoofSurface, which is not correct but it went unnoticed by our validation software (false negative) due to the angle of the normal. If the wall was modelled as a WallSurface it would be flagged as a semantically invalid surface, i.e. a false positive. It is likely that this dataset was converted to CityGML from another format, and during the conversion the surfaces have been semantically enriched according to orientation of the normals outwitting our validation procedure. Imagery (c) 2016 Microsoft Corp and Pictometry International Corp.

For this paper, we consider 5 classes of 9 of BoundarySurface types in LOD2, with respect to the conformance requirements in CityGML. The classes in LOD3 will include Window and Door, which cannot be validated only by normal, and have not been solved in our software. Validating the semantics is becoming an increasingly important task due to a growing number of semantically enriched datasets (Rook et al. 2016).

In short, we conclude that the validation of the geometry is a mostly solved problem, in contrast to the validation of semantics which presents a myriad of opportunities for future research. 


\section{CONCLUSION}

In this paper we have analysed numerous CityGML datasets from different sources to assess their quality and derive a list of prevalent errors. Our analysis shows that there is a large variation in the quality of CityGML datasets, and that each dataset is unique when it comes to errors. This is likely caused by different software packages which were used and different acquisition companies using different modelling guidelines resulting in different types of errors. None of the datasets we have tested was perfect, but some LOD1 datasets come very close to $100 \%$.

The non-planarity of polygons is by far the most common error we have encountered (error 203 in Figure 3). Other errors are less present, and we cannot say that there are omnipresent errors because the datasets are heterogeneous: while some datasets are full of a particular error, others are free of them. It should be noticed that this does not mean that errors of type $3 \times x$ (e.g. a shell having cracks and/or overlapping surfaces) do not appear, but since with our methodology all the primitives at one level must be valid to continue to the next one, then very often the validation of the properties of a solid is not attempted. Errors related to rings and polygons are thus more likely to be reported, but these are simpler to avoid and thus we were surprised that so many datasets have simple errors such as duplicate points or non-planarity.

In our opinion, most of the detected errors are easily preventable during the production of data. For 3D models that are automatically reconstructed, slight modifications to the algorithms used, e.g. triangulating non-planar faces or simply removing duplicate points, could have a big impact on the quality. For 3D models that are manually produced, it seems that the operators are often not aware of the quality rules used in the GIS world, and they produce their models by using Boolean operations such that their models look nice-if they were made aware of the rules then many of them could be easily avoided. However, this comes as no surprise because the quality of 3D city models is still an insufficiently explored topic, and the influence of particular errors onto specific spatial analyses has not been investigated.

Finally, it is important to note that we have carried out the validation entirely using our own software, which is released as opensource (under a GPL licence), so we hope that practitioners will use it to verify their datasets before releasing them for use.

\section{ACKNOWLEDGEMENTS}

We acknowledge the availability of the datasets from the following sources: Karlsruhe Institute of Technology, City of Berlin, District Administration Recklinghausen, Ordnance Survey Great Britain, City of the Hague, maila-push GmbH Darmstadt, GEORES, GTA Geoinformatik GmbH, City of Hamburg, University of Bonn, Landesvermessungsamt NRW, City of Linz, City of Lyon, City of Montreal, Technical University of Munich, Delft University of Technology, New York City Department of Information Technology and Telecommunications, City of Rotterdam, Singapore Land Authority, UVM Systems GmbH, CENSAM / Singapore-MIT Alliance for Research and Technology, Swiss Federal Office of Topography, Geneva's Territory Information System (SITG), and the City of Zürich.

This research is supported by (i) the Dutch Technology Foundation STW, which is part of the Netherlands Organisation for Scientific Research (NWO), and which is partly funded by the Ministry of Economic Affairs; (ii) China Scholarship Council (CSC); and (iii) Singapore Land Authority.

\section{References}

Agugiaro, G., 2016. First steps towards an integrated CityGML-based 3D model of Vienna. ISPRS Ann. Photogramm. Remote Sens. Spatial Inf. Sci. III-4, pp. 139-146.

Alam, N., Wagner, D., Wewetzer, M., Pries, M. and Coors, V., 2013. Towards automatic validation and healing of CityGML models for geometric and semantic consistency. ISPRS Ann. Photogramm. Remote Sens. Spatial Inf. Sci. II-2/W1, pp. 1-6.

Alam, N., Wagner, D., Wewetzer, M., von Falkenhausen, J., Coors, V. and Pries, M., 2014. Towards Automatic Validation and Healing of CityGML Models for Geometric and Semantic Consistency. In: Innovations in 3D Geo-Information Sciences, Springer International Publishing, pp. 77-91

Belussi, A., Migliorini, S., Negri, M. and Pelagatti, G., 2015. Validation of spatial integrity constraints in city models. In: Proceedings 4th ACM SIGSPATIAL International Workshop on Mobile Geographic Information Systems, ACM, pp. 70-79.

Biljecki, F., Heuvelink, G. B. M., Ledoux, H. and Stoter, J., 2015a. Propagation of positional error in 3D GIS: estimation of the solar irradiation of building roofs. International Journal of Geographical Information Science 29(12), pp. 2269-2294.

Biljecki, F., Ledoux, H. and Stoter, J., 2015b. Improving the consistency of multi-LOD CityGML datasets by removing redundancy. In: 3D Geoinformation Science, Springer International Publishing, pp. 1-17.

Biljecki, F., Ledoux, H. and Stoter, J., 2016. An improved LOD specification for 3D building models. Computers, Environment and Urban Systems 59, pp. 25-37.

Biljecki, F., Stoter, J., Ledoux, H., Zlatanova, S. and Çöltekin, A., 2015c. Applications of 3D city models: State of the art review. ISPRS International Journal of Geo-Information 4(4), pp. 2842-2889.

Boeters, R., Arroyo Ohori, K., Biljecki, F. and Zlatanova, S., 2015. Automatically enhancing CityGML LOD2 models with a corresponding indoor geometry. International Journal of Geographical Information Science 29(12), pp. 2248-2268

Bruse, M., Nouvel, R., Wate, P., Kraut, V. and Coors, V., 2015. An Energy-Related CityGML ADE and Its Application for Heating Demand Calculation. International Journal of 3-D Information Modeling 4(3), pp. 59-77.

Diakité, A. A., Damiand, G. and Gesquière, G., 2014. Automatic semantic labelling of 3D buildings based on geometric and topological information. In: Proc. 9th International 3DGeoInfo Conference, Dubai, United Arab Emirates, pp. 49-63.

Hsieh, C.-M., Aramaki, T. and Hanaki, K., 2011. Managing heat rejected from air conditioning systems to save energy and improve the microclimates of residential buildings. Computers, Environment and Urban Systems 35(5), pp. 358-367.

ISO, 2003. ISO 19107:2003: Geographic information-Spatial schema International Organization for Standardization.

Janssen, W. D., Blocken, B. and van Hooff, T., 2013. Pedestrian wind comfort around buildings: Comparison of wind comfort criteria based on whole-flow field data for a complex case study. Building and Environment 59, pp. 547-562

Kehl, C., 2015. Disseminating large-scale semantic 3D landscape models using open visualisation platforms. European Journal of Geography 6(2), pp. 51-68.

Ledoux, H., 2013. On the validation of solids represented with the international standards for geographic information. Computer-Aided Civil and Infrastructure Engineering 28(9), pp. 693-706.

Lee, D., Pietrzyk, P., Donkers, S., Liem, V., van Oostveen, J., Montazeri, S., Boeters, R., Colin, J., Kastendeuch, P., Nerry, F., Menenti, M., Gorte, B. and Verbree, E., 2013. Modeling and observation of heat losses from buildings: The impact of geometric detail on 3D heat flux modeling. In: Proceedings 33rd European Association of Remote Sensing Laboratories (EARSeL) Symposium, Matera, Italy, pp. 1-20. 
McKenney, D., 1998. Model quality: The key to CAD/CAM/CAE interoperability. Technical report, International TechneGroup Incorporated, Milford, $\mathrm{OH}$.

Mulder, D., 2015. Automatic repair of geometrically invalid 3D city building models using a voxel-based repair method. Master's thesis, 3D geoinformation group, Delft University of Technology, Netherlands.

Nouvel, R., Mastrucci, A., Leopold, U., Baume, O., Coors, V. and Eicker, U., 2015. Combining GIS-based statistical and engineering urban heat consumption models: Towards a new framework for multi-scale policy support. Energy and Buildings 107, pp. 204-212.

OGC, 2007. Geography markup language (GML) encoding standard. Open Geospatial Consortium inc. Document 07-036, version 3.2.1.

OGC, 2012. OGC city geography markup language (CityGML) encoding standard. Open Geospatial Consortium. Document 12-019, version 2.0.0.

OGC, 2016. OGC CityGML quality interoperability experiment. Open Geospatial Consortium. Document OGC 16-064.

Pédrinis, F., Morel, M. and Gesquière, G., 2015. Change Detection of Cities. In: 3D Geoinformation Science, Springer International Publishing, pp. 123-139.

Rook, M., Biljecki, F. and Diakité, A. A., 2016. Towards automatic semantic labelling of 3D city models. ISPRS Ann. Photogramm. Remote Sens. Spatial Inf. Sci

Salimzadeh, N., Sharif, S. A. and Hammad, A., 2016. Visualizing and Analyzing Urban Energy Consumption: A Critical Review and Case Study. In: Construction Research Congress 2016, American Society of Civil Engineers, Reston, VA, pp. 1323-1331.
Sindram, M., Machl, T., Steuer, H., Pültz, M. and Kolbe, T. H., 2016. Voluminator 2.0 - speeding up the approximation of the volume of defective 3D building models. ISPRS Ann. Photogramm. Remote Sens. Spatial Inf. Sci. III-2, pp. 29-36.

Stadler, A. and Kolbe, T. H., 2007. Spatio-semantic coherence in the integration of 3D city models. Int. Arch. Photogramm. Remote Sens. Spatial Inf. Sci. XXXVI-2/C43, pp. 1-8.

Steuer, H., Machl, T., Sindram, M., Liebel, L. and Kolbe, T. H., 2015. Voluminator-approximating the volume of 3D buildings to overcome topological errors. Springer, pp. 343-362.

Stoter, J., de Kluijver, H. and Kurakula, V., 2008. 3D noise mapping in urban areas. International Journal of Geographical Information Science 22(8), pp. 907-924.

Touya, G. and Brando-Escobar, C., 2013. Detecting Level-of-Detail Inconsistencies in Volunteered Geographic Information Data Sets. Cartographica: The International Journal for Geographic Information and Geovisualization 48(2), pp. 134-143.

van Walstijn, L., 2015. Requirements for an integral testing framework of CityGML instance documents. Master's thesis, Institute of Geodesy and Geoinformation Science, Technische Universitaet Berlin.

Zhao, J., Ledoux, H. and Stoter, J., 2013. Automatic repair of CityGML LoD2 buildings using shrink-wrapping. ISPRS Ann. Photogramm. Remote Sens. Spatial Inf. Sci. II-2/W1, pp. 309-317.

Zhao, J., Stoter, J. and Ledoux, H., 2014. A framework for the automatic geometric repair of CityGML models. In: Cartography from Pole to Pole, Springer, pp. 187-202. 